\title{
ENDOMORPHISM ALGEBRAS OF QM ABELIAN SURFACES
}

\author{
CHIA-FU YU
}

\begin{abstract}
We determine endomorphism algebras of abelian surfaces with quaternion multiplication.
\end{abstract}

\section{INTRODUCTION}

In this paper, we determine all possible endomorphism algebras of abelian surfaces with quaternion multiplication $(\mathrm{QM})$. Let $D$ be an indefinite quaternion division algebra over the field $\mathbb{Q}$ of rational numbers. We would like to find out all $\mathbb{Q}$-algebras $E$ containing $D$ which appear as endomorphism algebras of abelian surfaces. In other words, we would like to know which endomorphism algebra appears in the Shimura curve $X_{D}$ associated to the quaternion algebra $D$ (and with additional data). Our main result states as follows.

Theorem 1.1. Let $D$ be an indefinite quaternion division algebra over $\mathbb{Q}$, and let $A$ be an abelian surface over a field $k$ with quaternion multiplication by $D$, i.e. an abelian surface together with a $\mathbb{Q}$-algebra embedding $\iota: D \rightarrow E:=\operatorname{End}^{0}(A):=$ $\operatorname{End}(A) \otimes_{\mathbb{Z}} \mathbb{Q}$.

(1) Suppose that $A$ is not simple. Then $A$ is isogenous to $C^{2}$ for an elliptic curve $C$ over $k$ and the algebra $E$ is isomorphic to one of the following

(i) $\operatorname{Mat}_{2}(K)$, where $K$ is any imaginary quadratic field which splits $D$, or

(ii) $\operatorname{Mat}_{2}\left(D_{p, \infty}\right)$, where $p$ is a prime and $D_{p, \infty}$ is the quaternion algebra over $\mathbb{Q}$ ramified exactly at $\{p, \infty\}$. This occurs if and only if $C$ is a supersingular elliptic curve over $k$ with $k \supset \mathbb{F}_{p^{2}}$.

(2) Suppose that $A$ is simple. Then we have

(i) $E \simeq D$, or

(ii) $E \simeq D_{K}:=D \otimes_{\mathbb{Q}} K$ for some imaginary quadratic field $K$. In this case, the abelian surface $A$ is in characteristic $p>0$ for some prime $p$ and it is supersingular.

Recall that an abelian variety in characteristic $p>0$ is said to be supersingular if it is isogenous to a product of supersingular elliptic curves over a finite field extension. The case (i) of Theorem 1.1 (2) occurs as we can take a generic complex abelian surface with QM by $D$. The case (ii) of Theorem 1.1 (2) occurs only when the quaternion algebra $D$ satisfies a special condition and the simple abelian variety $A$ is necessarily supersingular. In this case, the algebra $E$ is obviously determined by its center $K$, and we show that there are only a finite list of possibilities for such $K$. More precisely, we have the following result.

Date: August 15, 2018.

2000 Mathematics Subject Classification. 11.

Key words and phrases. endomorphism algebras, QM abelian surfaces, quaternion algebras. 
Theorem 1.2 (Theorem 2.11). Let $A$ be a simple supersingular abelian surface over a finite field $\mathbb{F}_{q}$ of characteristic $p>0$ with quaternion multiplication by $D$. Let $E:=\operatorname{End}^{0}(A)$ be the endomorphism algebra of $A$, and let $S$ be the discriminant of $D$. Then

(1) The center $K$ of $E$ is isomorphic to $\mathbb{Q}\left(\zeta_{n}\right)$ for $n=3,4$, or 6 .

(2) One has $p \mid S$ and $p \equiv 1(\bmod n)$, where $n$ is as above, and for any other prime $\ell \mid S$, one has either $\ell \mid n$ or $\ell \equiv-1(\bmod n)$, that is, $\ell$ does not split in the quadratic field $\mathbb{Q}\left(\zeta_{n}\right)$.

(3) $E \simeq D \otimes_{\mathbb{Q}} K$.

According to Theorem 1.2, there are three possibilities for endomorphism algebras $E$ of simple supersingular abelian surfaces over finite fields: $E \simeq D \otimes_{\mathbb{Q}} \mathbb{Q}\left(\zeta_{n}\right)$ for $n=3,4,6$. However, not all of them occur; It depends on the quaternion algebra $D$. The algebra $D \otimes_{\mathbb{Q}} \mathbb{Q}\left(\zeta_{n}\right)$ occurs if and only if there is exactly one prime $p$ dividing $S$ such that $p \equiv 1(\bmod n)$ (This prime $p$ is the characteristic of the base field).

The results of this paper (Theorems 1.1 and 1.2) contribute new cases to the problem about semi-simple algebras that can be realized as endomorphism algebras of abelian varieties. See Oort 9 and the references therein for quite complete discussions of this problem.

Supersingular abelian surfaces appear in the classification of endomorphism algebras of QM abelian surfaces. We refer the reader to C. Xing [19] for some aspects of supersingular abelian surfaces over finite fields.

Let $J$ be the Jacobian of a smooth, projective, geometrically connected algebraic curve of genus 2 over a number field $K$. In [1] Baba and Granath showed that if the following three conditions hold: (1) $J$ has QM by a maximal quaternion order $\Lambda_{6}$ of discriminant $6,(2) J$ has the field of moduli equal to $\mathbb{Q}$, and (3) $J$ has potentially smooth stable reduction at both 2 and 3, then QM abelian surface $J$ has superspecial good reduction at infinitely many primes. In 4] Dieulefait and Rotger studied the arithmetic of the Jacobians $J$ whose endomorphism algebra $\operatorname{End} \frac{0}{\mathbb{Q}}(J)$ is an indefinite quaternion algebra. They determine all possible Galois groups of minimal fields of definition and possible endomorphism rings $\operatorname{End}_{K}(J)$ defined over a smaller number field $K$ under a certain integral condition. We refer the reader to 4 for the list of Galois groups and more detail discussions.

An analogous question to our main results (Theorems 1.1 and 1.2) is: What are the endomorphism algebras of abelian varieties with real multiplication (RM)? That is, one considers the same problem as treated in this paper but for Hilbert modular varieties rather than Shimura curves. This problem has been done by Chai [3, Section 3]. The classification has its own interest; this also plays a role in the proof of Chai's theorem on the density of ordinary Hecke orbits in Siegel modular varieties. As the reader may be also interested in this result due to Chai, we include an expository and elementary account for the reader's convenience in Section 3. Using the similar method as in Section 2 we make Chai's result more explicit about the simple algebras that actually occur as endomorphism algebras of RM abelian varieties.

\section{Proof of Main Results}


2.1. Embeddings of simple algebras. We recall some basic definitions for central simple algebras; see [11.

Definition 2.1. Let $B$ be a (f.d.) central simple algebra over a field $F$. The degree, capacity, and index of $B$ are defined as

$$
\operatorname{deg}(B):=\sqrt{[B: F]}, \quad \mathrm{c}(B):=n, \quad \mathrm{i}(B):=\sqrt{[\Delta: F]},
$$

respectively, if $B \cong \operatorname{Mat}_{n}(\Delta)$, where $\Delta$ is a division algebra over $F$, which is uniquely determined by $B$ up to isomorphism. The algebra $\Delta$ is also called the division part of $B$.

Proposition 2.2. Let $E$ and $B$ be two finite-dimensional simple algebras over a field $F$ with centers $Z$ and $K$, respectively. Suppose that $Z$ and $K$ are linearly disjoint over $F$, that is, the F-algebra $L:=Z \otimes_{F} K$ is a field. Let $E \simeq \operatorname{Mat}_{n}(\Delta)$, where $\Delta$ is the division part of $E$. Then there is an $F$-algebra embedding of $B$ into $E$ if and only if

$$
[B: F] \mid n \cdot c,
$$

where $c$ is the capacity of the central simple algebra $\Delta \otimes_{F} B^{\circ}$ over $L$ :

$$
\Delta \otimes_{F} B^{\circ} \simeq \Delta \otimes_{Z}\left(Z \otimes_{F} K\right) \otimes_{K} B^{\circ} \simeq\left(\Delta \otimes_{Z} L\right) \otimes_{L}\left(L \otimes_{K} B^{\circ}\right),
$$

and $B^{\circ}$ denotes the opposite algebra of $B$.

Proof. This is a special case of [18, Theorem 1.2]. However, instead of referring to the general result, we prefer to give a direct proof for the reader's convenience. Let $E=\operatorname{End}_{\Delta}(V)$, where $V$ is a right vector space over $\Delta$. An $F$-algebra embedding from $B$ into $E$ exists if and only if $V$ is a $(B, \Delta)$-bimodule, or equivalently a right $\Delta \otimes_{F} B^{\circ}$-module. Let $\Delta \otimes_{F} B^{\circ} \simeq \operatorname{Mat}_{c}\left(\Delta^{\prime}\right)$, where $\Delta^{\prime}$ is the division part of the simple algebra $\Delta \otimes_{F} B^{\circ}$. By the dimension counting, the vector space $V$ is a $\operatorname{Mat}_{c}\left(\Delta^{\prime}\right)$-module if and only if

$$
\frac{\operatorname{dim}_{F} V}{c\left[\Delta^{\prime}: F\right]} \in \mathbb{N}
$$

Note that $[B: F][\Delta: F]=c^{2}\left[\Delta^{\prime}: F\right]$. From this relation and that $\operatorname{dim}_{F} V=n[\Delta$ : $F]$, the condition (2.2) can be written as $[B: F] \mid n c$. This proves the proposition.

Remark 2.3. The reader can find in [18 for more general results about Proposition 2.2 where $B$ and $E$ are any finite-dimensional semi-simple $F$-algebras. When $F$ is a global field, the local-global principle enters and plays a role in the problem of embeddings of simple algebras. For a detailed discussion, the reader is referred to the paper [12.

After establishing a basic embedding result (Proposition 2.2), we begin with the classification of endomorphism algebras of QM abelian surfaces. Let $(A, \iota)$ be an abelian surface with quaternion multiplication by $D$ and let $E:=\operatorname{End}^{0}(A):=$ $\operatorname{End}(A) \otimes_{\mathbb{Z}} \mathbb{Q}$ be the endomorphism algebra of $A$. 
2.2. Case where $A$ is not simple. In this case $A$ is isogenous to $C_{1} \times C_{2}$, where $C_{1}$ and $C_{2}$ are elliptic curves. Then $C_{1}$ is isogenous to $C_{2}$. If not, then we have inclusions $D \subset \operatorname{End}^{0}\left(C_{i}\right)$ for $i=1,2$ and each $C_{i}$ must be supersingular. It follows that $D \simeq \operatorname{End}^{0}\left(C_{i}\right) \simeq D_{p, \infty}$, the definite quaternion algebra over $\mathbb{Q}$ ramified exactly at $\{p, \infty\}$, contradiction.

Therefore, the algebra $E=\operatorname{End}^{0}(A)=\operatorname{End}(A) \otimes \mathbb{Q}$ is isomorphic to one the following:

(i) $\operatorname{Mat}_{2}(\mathbb{Q})$,

(ii) $\operatorname{Mat}_{2}(K)$, where $K$ is an imaginary quadratic field,

(iii) $\operatorname{Mat}_{2}\left(D_{p, \infty}\right)$.

The case $(\mathrm{i})$ can not occur because $D$ and $\operatorname{Mat}_{2}(\mathbb{Q})$ are different quaternion algebras.

The case (ii) can appear if and only if $K$ splits $D$. Indeed, as one has an embedding of $D$ in $\operatorname{Mat}_{2}(K)$, the algebra $D$ acts on a $K$-vector space $V$ of dimension two. We can identity $V$ with $D$ as $V$ is one-dimensional $D$-vector space. This makes $D$ a $K$-vector space of dimension 2 . Therefore, $K$ is isomorphic to a (necessarily) maximal subfield of $D$. This is exactly when $K$ splits $D$. On the other hand, any imaginary quadratic field is isomorphic to the endomorphism algebra of an elliptic curve. Therefore, for any imaginary quadratic field that splits $D$, the matrix algebra $\mathrm{Mat}_{2}(K)$ can occur as the endomorphism algebra of a QM abelian surface.

For the case (iii), this occurs of course only when $A$ is in characteristic $p>0$ and $A$ is isogenous to the product of two supersingular elliptic curves over the base field $k$ containing $\mathbb{F}_{p^{2}}$. Now we check that an embedding $\iota: D \rightarrow \operatorname{Mat}_{2}\left(D_{p, \infty}\right)$ exists for any prime $p$. By Proposition 2.2 , we need to show that $[D: \mathbb{Q}] \mid 2 c$, where $c$ is the capacity of the central simple algebra $D^{\circ} \otimes_{\mathbb{Q}} D_{p, \infty}$ over $\mathbb{Q}$ and $D^{\circ}$ denotes the opposite algebra of $D$. As the tensor product of two quaternion algebras is Brauer equivalent to a quaternion algebra, we have

$$
D^{\mathrm{o}} \otimes_{\mathbb{Q}} D_{p, \infty} \simeq \operatorname{Mat}_{2}\left(D^{\prime}\right)
$$

for some definite quaternion algebra $D^{\prime}$ over $\mathbb{Q}$. This shows that $c=2$ and hence that a $\mathbb{Q}$-algebra embedding $\iota: D \rightarrow \operatorname{Mat}_{2}\left(D_{p, \infty}\right)$ exists. We have shown the following result.

Proposition 2.4. Let $D$ be as above and $A$ be an abelian surface with endomorphism algebra $E:=\operatorname{End}^{0}(A)$ containing $D$. Suppose that $A$ is not simple. Then $A$ is isogenous to $C^{2}$ for an elliptic curve $C$ and the algebra $E$ is isomorphic to one of the following two cases

(i) $\operatorname{Mat}_{2}(K)$, where $K$ is any imaginary quadratic field which splits $D$, or

(ii) $\operatorname{Mat}_{2}\left(D_{p, \infty}\right)$. This occurs if and only if $C$ is a supersingular elliptic curve in characteristic $p>0$ and the base field $k$ contains $\mathbb{F}_{p^{2}}$.

2.3. Case where $A$ is simple. Since $E:=\operatorname{End}^{0}(A)$ contains the quaternion algebra $D$, the algebra $E$ is non-commutative. Let $K$ be the center of $E$. Since $\operatorname{dim} A=2$, any maximal subfield of $E$ has degree 2 or 4 (over $\mathbb{Q}$ ). So one has $[K: \mathbb{Q}] \mid 4$. If $[K: \mathbb{Q}]=4$, then $E=K$ (which is commutative), absurd. So $K=\mathbb{Q}$ or $[K: \mathbb{Q}]=2$. 
If $K=\mathbb{Q}$, which is totally real, then $E$ is a quaternion algebra over $\mathbb{Q}$. This follows from Albert's classification of central division algebras with positive involution (cf. Mumford [6, Section 21]). In this case, one must have $E \simeq D$.

Suppose now that $[K: \mathbb{Q}]=2$. Then $E$ is a quaternion division algebra over $K$. If $K$ is real, then $E \supset D \otimes_{\mathbb{Q}} K$ contains a totally real maximal subfield $K^{\prime}$ (of degree 4 over $\mathbb{Q}$ ), which shows that $\operatorname{dim} A$ is divisible by 4 (see Mumford [6, Corollary, p. 191]), absurd. It follows that the center $K$ is an imaginary quadratic field. Note that in this case, $A$ is in characteristic $p>0$ for some prime $p$. Indeed, its endomorphism algebra contains a 4-dimensional CM subfield We also know that any simple $\mathrm{CM}$ abelian variety by a $\mathrm{CM}$ field $L$ in characteristic zero has endomorphism algebra equal to $L$ but $E$ is non-commutative. Therefore, $A$ is in positive characteristic. Now we determine which quaternion division algebra $E$ over an imaginary quadratic field $K$ contains a subalgebra isomorphic to $D$. This is exactly when the capacity of $E \otimes_{\mathbb{Q}} D^{\circ}$ is equal to 4 by Proposition 2.2, or equivalently, the quaternion algebra $D_{K}:=D \otimes_{\mathbb{Q}} K$ is isomorphic to $E$. We have shown the following result.

Proposition 2.5. Let $D$ be as above and $A$ be an abelian surface with quaternion multiplication by $D$. Suppose that $A$ is simple. Then

(i) $E \simeq D$, or

(ii) $E \simeq D_{K}:=D \otimes_{\mathbb{Q}} K$ for some imaginary quadratic field $K$. In this case, the abelian surface $A$ is in characteristic $p>0$ for some prime $p$.

Theorem 1.1 follows from Propositions 2.4 and 2.5 .

The case (i) of Proposition 2.5 occurs as one can take $A$ to be a generic complex QM abelian surface. For the case (ii), we make a further discussion about the algebras of the form $D_{K}$ that can occur in the next subsection.

2.4. Put $D_{K}:=D \otimes_{\mathbb{Q}} K$, where $K$ is an imaginary quadratic field. In the remaining of this section, we investigate which $D_{K}$ can be realized as the endomorphism algebra of an abelian surface.

Suppose $D_{K} \simeq \operatorname{End}^{0}(A)$ for an abelian surface. Then $A$ has smCM (sufficiently many complex multiplications, that is, the endomorphism algebra $\operatorname{End}^{0}(A)$ of $A$ contains a semi-simple commutative $\mathbb{Q}$-subalgebra $L$ with $[L: \mathbb{Q}]=2 \operatorname{dim} A$ ). By a theorem of Grothendieck [6. Section 22, p. 220], there are a finite field extension $k^{\prime}$ of the ground field $k$ and an abelian surface $A_{0}$ over a finite field $k_{0}$ contained in $k^{\prime}$ such that there is an isogeny $A \otimes_{k} k^{\prime} \rightarrow A_{0} \otimes_{k_{0}} k^{\prime}$ over $k^{\prime}$ (see [7] for Grothendieck's original proof and [16] for a different proof). We may enlarge $k_{0}$ in $k^{\prime}$, if necessary, such that $\operatorname{End}^{0}\left(A_{0} \otimes_{k_{0}} k^{\prime}\right)=\operatorname{End}_{k_{0}}^{0}\left(A_{0}\right)=: E_{0}$. This shows the following:

Lemma 2.6. Notations being as above, the algebra $E \simeq D_{K}$ is contained in the endomorphism algebra $E_{0}$ of an abelian surface over a finite field.

Since $[E: \mathbb{Q}]=8$, one has either

(a) $E=E_{0}$, or

(b) $\operatorname{dim} E_{0}=16$.

Lemma 2.7. Let notations be as above. For either the case (a) or (b), there is a rational prime $p$ which splits in $K$. Furthermore we have for any finite place $v$ of 


$$
\operatorname{inv}_{v}(E)= \begin{cases}1 / 2 & \text { if } v \mid p, \\ 0 & \text { otherwise. }\end{cases}
$$

Proof. For the case (b), the algebra $E_{0} \simeq \operatorname{Mat}_{2}\left(D_{p, \infty}\right)$ for some rational prime $p$. The algebra $D_{K}$ can be embedded in $E_{0}$ if and only if $D_{p, \infty} \otimes_{\mathbb{Q}} K \simeq D_{K} \simeq E$. Since $\operatorname{inv}_{\infty}(E)=0$ and $E$ is a division algebra, the places with non-trivial invariants are those of $K$ over $p$. It follows that there are two places of $K$ lying over $p$ at which $E$ has non-trivial local invariant, and the remaining local invariants are trivial.

For the case (a), $E$ is the endomorphism algebra of an abelian surface $A_{0}$ over a finite field $k_{0}$. Using the Honda-Tate theory, the center $K$ is $\mathbb{Q}\left(\pi_{0}\right)$, where $\pi_{0}$ is the relative Frobenius endomorphism of $A_{0}$ over $k_{0}$. For any finite place $v$ of $K$ with $v \nmid p$, one has $\operatorname{inv}_{v}(E)=0$. As $E$ is a division algebra, it follows that there are two places of $K$ lying over $p$ at which $E$ has non-trivial local invariant, and the remaining local invariants are trivial.

We need to find all rational primes $p$ and imaginary quadratic fields $K$ such that the quaternion algebra $D_{K}:=D \otimes_{\mathbb{Q}} K \simeq E$ satisfies the condition of Lemma 2.7 and that the algebra $E$ appears as the endomorphism algebra of an abelian surface. Let $S$ be the discriminant of $D$ over $\mathbb{Q}$; by definition, $S$ is the product of all finite ramified rational primes for $D$. Clearly, one has $p \mid S$, otherwise the local invariants of $D_{K}$ at places $v$ lying over $p$ are zero and hence that $D_{K} \simeq \operatorname{Mat}_{2}(K)$, absurd. Therefore, a necessary condition that $K$ satisfies the conditions in Lemma 2.7 is the following:

(*) The prime $p$ splits in $K$ and for any other prime $\ell \mid S$, the completion $K_{\ell}:=$ $K \otimes \mathbb{Q}_{\ell}$ at $\ell$ is a field.

The first condition of $(*)$ follows from $\operatorname{inv}_{v}(E)=1 / 2$ if $v \mid p$ and the second one follows from $\operatorname{inv}_{v}(E)=0$ otherwise.

Now given a rational prime $p \mid S$ and an imaginary quadratic field $K$ satisfying the condition $(*)$, we would like to find a Weil $q$-number $\pi$, where $q$ is a power of $p$, so that $K \simeq \mathbb{Q}(\pi)$ and for every place $v \mid p$ of $K$, one has $v(\pi) / v(q)=1 / 2$.

Lemma 2.8. Let $(A, \iota)$ be an abelian surface with $Q M$ by $D$ over a field $k$ of characteristic $p>0$. Suppose that $p \mid S$, then $A$ is supersingular.

Proof. This result is well-known (see [2]); we provide a proof for the reader's convenience. We may assume that the ground field $k$ is algebraically closed. We have a $\mathbb{Q}_{p}$-algebra embedding $\iota: D_{p}:=D \otimes \mathbb{Q}_{p} \rightarrow \operatorname{End}^{0}(X)$, where $X:=A\left[p^{\infty}\right]$ is the $p$-divisible group attached to $A$. The possibilities of $\operatorname{End}^{0}(X)$ are (a) (ordinary) $\operatorname{Mat}_{2}\left(\mathbb{Q}_{p}\right) \times \operatorname{Mat}_{2}\left(\mathbb{Q}_{p}\right)$, (b) ( $p$-rank one) $D_{p} \times \mathbb{Q}_{p} \times \mathbb{Q}_{p}$, and (c) (supersingular) $\operatorname{Mat}_{2}\left(D_{p}\right)$. Clearly, only the case (c) is possible. Therefore, the abelian surface $A$ is supersingular.

We need to find all Weil $q$-numbers $\pi$ so that the corresponding abelian variety $A_{\pi}$, uniquely determined up to isogeny, is both simple and supersingular, and that its center $\mathbb{Q}(\pi)$ is an imaginary quadratic field satisfying the condition $(*)$. The latter condition will imply that the endomorphism algebra of $A_{\pi}$ is a quaternion division algebra over $K$ and hence that $A_{\pi}$ is an abelian surface. 
Theorem 2.9. Let $q$ be a power of a prime number $p$ and $\pi$ is a Weil q-number. Then the corresponding abelian variety $A_{\pi}$ is supersingular if and only if $\pi=\sqrt{q} \zeta$, where $\zeta$ is a root of unity.

Proof. This is a well-known immediate consequence of results due to Manin [5, Tate [14] and Oort [8, Theorem 2], also see [19]. We provide a proof for the reader's convenience. Let $C$ be a supersingular elliptic curve over $\mathbb{F}_{p}$ such that $\pi_{C}^{2}+p=0$, where $\pi_{C}$ is the Frobenius endomorphism of $C / \mathbb{F}_{p}$. Put $A_{1}=C^{g}$, where $g=\operatorname{dim} A_{\pi}$. Since any two supersingular abelian varieties are isogenous over a finite extension of their ground fields, we have $\pi^{N}=p^{M}$ for some positive integers $N$ and $M$. It follows that $\pi$ is of the form $\sqrt{q} \zeta$, where $\zeta$ is a root of unity. Conversely, suppose that $\pi$ is of this form. Then $\pi^{N}$, for some even integer $N$, is $q^{N / 2}$, which is a Weil number corresponding to a supersingular elliptic curve. By Tate's isogeny theorem, $A$ is isogenous to the product of copies of a supersingular elliptic curve over a finite field. This completes the proof of the theorem.

We shall call a Weil $q$-number $\pi$ supersingular if the corresponding simple abelian variety $A_{\pi}$ up to isogeny is supersingular.

Lemma 2.10. Let $\pi=\sqrt{q} \zeta_{n}$ be a supersingular Weil q-number, where $q=p^{a}$, and $\zeta_{n}$ is a primitive $n$-th root of unity. Then the field $K=\mathbb{Q}(\pi)$ generated by $\pi$ is an imaginary quadratic field if and only if

(a) $a$ is even and $n=3,4,6$, or

(b) $a$ is odd and $n=4, n=8$ and $p=2$, or $n=12$ and $p=3$.

Proof. (a) One has $\mathbb{Q}(\pi)=\mathbb{Q}\left(\zeta_{n}\right)$, so $[\mathbb{Q}(\pi): \mathbb{Q}]=2$ if and only if $n=3,4,6$. (b) We have $\mathbb{Q}(\pi)=\mathbb{Q}\left(\sqrt{p} \zeta_{n}\right)$. Since $\mathbb{Q}\left(\pi^{2}\right)=\mathbb{Q}\left(\zeta_{m}\right)$ has degree one or two, $m=2,3,4$ or 6 and $n=4,3,6,8$ or 12 . The case $n=4$ is good. For the remaining cases one must have $\mathbb{Q}(\pi)=\mathbb{Q}\left(\pi^{2}\right)$. Note that 2 is the only ramified prime in $\mathbb{Q}\left(\zeta_{4}\right)$ and 3 is the only ramified prime in $\mathbb{Q}\left(\zeta_{3}\right)=\mathbb{Q}\left(\zeta_{6}\right)$. Since $p$ is ramified in $\mathbb{Q}(\pi)$, the only possibilities are $\mathbb{Q}\left(\sqrt{2} \zeta_{8}\right)$ and $\mathbb{Q}\left(\sqrt{3} \zeta_{n}\right)$ for $n=3,6,12$. It is easy to check that only $\mathbb{Q}\left(\sqrt{2} \zeta_{8}\right)$ and $\mathbb{Q}\left(\sqrt{3} \zeta_{12}\right)$ are quadratic fields.

Note that in the case (a) the prime $p$ splits in $\mathbb{Q}(\pi)$ if and only if $p \equiv 1(\bmod n)$. In the case (b) $p$ is ramified in $\mathbb{Q}(\pi)$. Therefore, if one requires the field $\mathbb{Q}(\pi)$ satisfy the condition $(*)$, then only the case (a) can occur. This yields the following conclusion.

Theorem 2.11. Let $A$ be a simple supersingular abelian surface over a finite field $\mathbb{F}_{q}$ of characteristic $p>0$ with quaternion multiplication by $D$ and let $E:=$ $\operatorname{End}^{0}(A)$. Then

(1) The center $K$ of $E$ is isomorphic to $\mathbb{Q}\left(\zeta_{n}\right)$ for $n=3,4$, or 6 .

(2) One has $p \mid S$ and $p \equiv 1(\bmod n)$, where $n$ is as above, and for any other prime $\ell \mid S$, one has either $\ell \mid n$ or $\ell \equiv-1(\bmod n)$.

(3) $E \simeq D \otimes_{\mathbb{Q}} K$.

It follows from Theorem 2.11 that there are three possibilities for endomorphism algebras $E$ of simple supersingular abelian surfaces over finite fields: $E \simeq D \otimes_{\mathbb{Q}}$ $\mathbb{Q}\left(\zeta_{n}\right)$ for $n=3,4,6$. Furthermore, the algebra $D \otimes \mathbb{Q} \mathbb{Q}\left(\zeta_{n}\right)$ occurs if and only if there is exactly one prime $p \mid S$ such that $p \equiv 1(\bmod n)$. This prime is the characteristic of the ground field of the abelian surface. 
We conclude this section with a remark and a question we think interesting. Let $E$ be the endomorphism algebra of a simple QM abelian surface $A$ such that $E \neq D$. Then

- $A$ is in characteristic $p>0$ for a prime $p$, and $A$ is supersingular,

- $E=D \otimes_{\mathbb{Q}} K$, where $K$ is an imaginary quadratic field satisfying the condition $(*)$ after Lemma 2.7.

- In case that $E$ is isomorphic to the endomorphism algebra of supersingular simple QM abelian surface over a finite field, we know all such $K$ that can occur by Theorem 2.11,

However, we are not able to rule out the possibility that $E$ is not isomorphic to a (necessarily supersingular) simple QM abelian surface over a finite field. That is, whether or not the endomorphism algebra of any supersingular abelian surface over an arbitrary field $k$ is isomorphic to that of one over a finite field. We make the following hypothesis.

(H) Let $k, k^{\prime}$ and $k_{0}$ be three fields with the inclusion relation $k \subset k^{\prime} \supset k_{0}$. Let $A / k$ and $A_{0} / k_{0}$ be two abelian varieties such that there is an isogeny $\varphi$ : $A \otimes_{k} k^{\prime} \rightarrow A_{0} \otimes_{k_{0}} k^{\prime}$ over $k^{\prime}$. Suppose that $\operatorname{End}^{0}\left(A_{0}\right)=\operatorname{End}^{0}\left(A_{0} \otimes_{k_{0}} k^{\prime}\right)$. We identify $\operatorname{End}^{0}\left(A \otimes_{k} k^{\prime}\right)=\operatorname{End}^{0}\left(A_{0}\right)$ using the isogeny $\varphi$. Then there an abelian variety $A_{1} / k_{1}$ over a subfield $k_{1} \subset k_{0}$ and an isogeny $\varphi_{1}: A_{1} \otimes_{k_{1}} k_{0} \rightarrow A_{0}$ over $k_{0}$ so that the subalgebra $\operatorname{End}^{0}\left(A_{1}\right) \subset \operatorname{End}^{0}\left(A_{0}\right)$ (through $\varphi_{1}$ ) is equal to the subalgebra $\operatorname{End}^{0}(A) \operatorname{in} \operatorname{End}^{0}\left(A \otimes_{k} k^{\prime}\right)=\operatorname{End}^{0}\left(A_{0}\right)$.

The hypothesis $(\mathbf{H})$ rules out the possibility of imaginary quadratic fields $K$ satisfying the necessary condition $(*)$ that are not of the shape described in Theorem [2.11. Then Theorems 1.1 and 1.2 give a complete result for endomorphism algebras of QM abelian surfaces over an arbitrary base field. Besides, we also obtain the following result, which is an immediate consequence of a theorem of Grothendieck (cf. [7, [16]).

Corollary 2.12. Let $A / k$ be an abelian variety that has smCM over a field $k$ of characteristic $p>0$. Assume the hypothesis $(\mathbf{H})$. Then the endomorphism algebra $\operatorname{End}^{0}(A)$ of $A / k$ is isomorphic to that of an abelian variety over a finite field.

Even when the hypothesis $(\mathbf{H})$ fails, one is still in an interesting situation. This means there are some subtle issues about the fields of definition that we were not aware of. For example, there are endomorphism algebras of abelian varieties having smCM in positive characteristic that can not be found by the Honda-Tate theory. These would contribute new examples to the problem of endomorphism algebras of abelian varieties studied in Oort 9].

The following question should be helpful to understand the problem of fields of definition arising from $(\mathbf{H})$. Recall that an abelian variety over a field $k$ of characteristic $p>0$ is said to be superspecial if it is isomorphic to a product of supersingular elliptic curves over an algebraic closure of $k$.

(Q). Let $A$ be a superspecial abelian variety over a field $k$ of characteristic $p>0$. Is there a superspecial abelian variety $A_{0}$ over a finite field $k_{0}$ so that $A$ is isomorphic to $A_{0} \otimes_{k_{0}} k$ over $k$ ? 


\section{ENDOMORPHISM ALGEBRAS OF RM ABELIAN VARIETIES}

In this section, we give an exposition on endomorphism algebras of abelian varieties with real multiplication. Our reference is Chai [3], especially Section 3 of it. The classification has its own interest; this is also useful in the proof of Chai's theorem on the density of ordinary Hecke orbits in Siegel modular varieties. We change the notations a bit. Let $F$ be a totally real number field of degree $g=[F: \mathbb{Q}]$, and let $O_{F}$ be the ring of integers. An abelian variety with real multiplication by $O_{F}$ is a pair $(A, \iota)$, where $A$ is a $g$-dimensional abelian variety and $\iota: O_{F} \rightarrow \operatorname{End}(A)$ is a ring monomorphism. As we are only concerned with the endomorphism algebra $\operatorname{End}^{0}(A)$ of such objects, we may replace the $\operatorname{ring}$ monomorphism $\iota: O_{F} \rightarrow \operatorname{End}(A)$ by its induced $\mathbb{Q}$-algebra embedding $\iota: F \rightarrow \operatorname{End}^{0}(A)$. We shall call the latter object $(A, \iota)$ an abelian variety with $R M$ by $F$.

Let $(A, \iota)$ be an abelian variety with RM by $F$ over an (unspecified) base field $k$.

Lemma 3.1. The underlying abelian variety $A$ is isogenous to $A_{1}^{n}$, where $A_{1}$ is a simple abelian variety.

Proof. Let $A$ be isogenous to $\prod_{j=1}^{r} A_{j}^{n_{j}}$, where $A_{i}$ and $A_{j}$ are non-isogenous simple abelian varieties if $i \neq j$. Then one has a $\mathbb{Q}$-algebra embedding $\iota: F \rightarrow$ $\operatorname{End}^{0}\left(A_{j}^{n_{j}}\right)$ for each $j=1, \ldots, r$, and hence $g \mid \operatorname{dim} A_{j}^{n_{j}}$. It follows that $r=1$.

Put $d:=\operatorname{dim} A_{1}$ and $\Delta:=\operatorname{End}^{0}\left(A_{1}\right)$. One has $g=n d$ and $\operatorname{End}^{0}(A)=\operatorname{Mat}_{n}(\Delta)$. The division algebra $\Delta$ admits a positive (Rosati) involution $*$. We use the classification of Albert for $\Delta$ (cf. [6, Section 21]).

(Type I) The algebra $\Delta=K_{0}$ is a totally real number field. Since $F \simeq \iota(F) \subset$ $\operatorname{Mat}_{n}\left(K_{0}\right)$, one has $g \mid n\left[K_{0}: \mathbb{Q}\right]$. On the other hand, one has $\left[K_{0}: \mathbb{Q}\right] \mid d$. It follows that $d=\left[K_{0}: \mathbb{Q}\right]$. The map $\iota$ makes the $K_{0}$-vector space $V=K_{0}^{n}$ as an $F$-vector space of dimension one. Therefore, $F$ can be regraded as a $K_{0}$-vector space and hence $K_{0}$ is isomorphic to a subfield of $F$. We may assume that $K_{0}$ is a subfield of $F$. Conversely, given a subfield $K_{0}$ of $F$ of degree $d$, we choose an abelian variety $A_{1}$ so that the endomorphism algebra $\operatorname{End}^{0}\left(A_{1}\right)$ is isomorphic to $K_{0}$. Take $A:=A_{1}^{n}$, where $n:=g / d$. Since there is a $\mathbb{Q}$-algebra embedding $\iota: F \rightarrow \operatorname{Mat}_{n}\left(K_{0}\right)$, we have an abelian variety $(A, \iota)$ with $\mathrm{RM}$ by $F$ such that $\operatorname{End}^{0}(A)$ is isomorphic to $\operatorname{Mat}_{n}\left(K_{0}\right)$.

(Type II) The algebra $\Delta$ is a totally indefinite quaternion algebra over a totally real number field $K_{0}$. Since $\Delta$ contains a totally real maximal subfield $K_{1}$, which has degree $2\left[K_{0}: \mathbb{Q}\right]$, one has $2\left[K_{0}: \mathbb{Q}\right] \mid \operatorname{dim} A_{1}$. On the other hand, any maximal subfield of $\operatorname{Mat}_{n}(\Delta)$ has degree $2 n\left[K_{0}: \mathbb{Q}\right]$, which gives the other divisibility $g$ $2 n\left[K_{0}: \mathbb{Q}\right]$. Therefore, we have $g=2 n\left[K_{0}: \mathbb{Q}\right]$ and $\operatorname{dim} A_{1}=2\left[K_{0}: \mathbb{Q}\right]$. Since $F$ has the degree of maximal semi-simple commutative subalgebras of $\operatorname{Mat}_{n}(\Delta)$, the image of any embedding $\iota$ contains the center $K_{0}$. Therefore, we may assume that $K_{0}$ is a subfield of $F$ and $\iota$ is a $K_{0}$-algebra embedding of $F$ into $\operatorname{Mat}_{n}(\Delta)$. The field $F$ is isomorphic to a (maximal) subfield of $\operatorname{Mat}_{n}(\Delta)$ if and only if $F$ splits $\Delta$. The latter is equivalent to that for any place $v \in \operatorname{Ram}\left(\Delta / K_{0}\right)$, the set of ramified places of $K_{0}$ for $\Delta$, and any place $w \mid v$ of $F$, one has $\left[F_{w}: K_{0, v}\right] \equiv 0(\bmod 2)$. 
Conversely, suppose we have a subfield $K_{0}$ of $F$ with $2\left[K_{0}: \mathbb{Q}\right] n=g$ for some positive integer $n$, and a totally indefinite quaternion algebra $\Delta$ over $K_{0}$ which is split by $F$. Then there exists an abelian variety with RM by $F$ so that $\operatorname{End}^{0}(A) \simeq \operatorname{Mat}_{n}(\Delta)$. Indeed, we first take a complex abelian variety $A_{1}$ with dimension $2\left[K_{0}: \mathbb{Q}\right]$ so that $\operatorname{End}^{0}\left(A_{1}\right) \simeq \Delta$. Then put $A:=A_{1}^{n}$. The condition that $F$ splits $\Delta$ implies that there is a $K_{0}$-algebra embedding from $F$ into $\operatorname{Mat}_{n}(\Delta)$. This way we construct a (complex) abelian variety with RM by $F$ so that $\operatorname{End}^{0}(A) \simeq \operatorname{Mat}_{n}(\Delta)$.

(Type III) The algebra $\Delta$ is a totally definite quaternion algebra over a totally real number field $K_{0}$. Since $F$ can be embedded in $\operatorname{Mat}_{n}(\Delta)$, whose maximal semi-simple commutative subalgebras have the same degree $2 n\left[K_{0}: \mathbb{Q}\right]$, one has $g \mid 2 n\left[K_{0}: \mathbb{Q}\right]$. On the other hand, as the field $K_{0}$ acts on the abelian variety $A_{1}$ up to isogeny, one has $\left[K_{0}: \mathbb{Q}\right] \mid \operatorname{dim} A_{1}$; this gives the condition $n\left[K_{0}: \mathbb{Q}\right] \mid g$. Also, if the ground field $k$ is of characteristic zero, then the algebra $\Delta$ acts on the homology group $H_{1}\left(A_{1}, \mathbb{Q}\right)$. This gives the condition $4\left[K_{0}: \mathbb{Q}\right] \mid 2 \operatorname{dim} A_{1}$, or equivalently $2 n\left[K_{0}: \mathbb{Q}\right] \mid g$. We have two cases:

(a) $g=n\left[K_{0}: \mathbb{Q}\right]$. This case occurs only when $k$ is of characteristic $p>0$ for some prime $p$.

(b) $g=2 n\left[K_{0}: \mathbb{Q}\right]$.

We first rule out the possibility of (b). Since $g$ is the degree of any maximal semisimple commutative subalgebra of $\operatorname{Mat}_{n}(\Delta)$, the image of $F$ under any embedding $\iota: F \rightarrow \operatorname{Mat}_{n}(\Delta)$ contains the center $K_{0}$. Therefore, $F$ contains a subfield which is isomorphic to $K_{0}$, and we may assume that the field $F$ contains $K_{0}$ and the

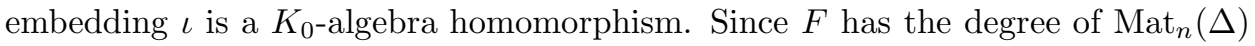
over $K_{0}$, the field $F$ can be embedded into the simple algebra $\operatorname{Mat}_{n}(\Delta)$ if and only if $F$ splits $\Delta$. But the latter is impossible because $\Delta$ is totally definite and $F$ is totally real.

For the case $(\mathrm{a})$, we have $\operatorname{dim} A_{1}=\left[K_{0}: \mathbb{Q}\right]$. In this case the abelian variety $A_{1}$ has smCM. By a theorem of Grothendieck [6, Section 22, p. 220], there are a finite field extension $k^{\prime} / k$, an abelian variety $A_{0}$ over a finite field $k_{0}$ contained in $k^{\prime}$ and an isogeny $\varphi: A_{1} \otimes_{k} k^{\prime} \rightarrow A_{0} \otimes_{k_{0}} k^{\prime}$. Enlarging $k_{0}$ if necessary, we can assume that $\operatorname{End}^{0}\left(A_{0} \otimes_{k_{0}} k^{\prime}\right)=\operatorname{End}^{0}\left(A_{0}\right)$. We have

$$
\Delta=\operatorname{End}^{0}\left(A_{1}\right) \subset \operatorname{End}^{0}\left(A_{1} \otimes_{k} k^{\prime}\right) \simeq \operatorname{End}^{0}\left(A_{0} \otimes_{k_{0}} k^{\prime}\right)=\operatorname{End}^{0}\left(A_{0}\right) .
$$

We first show that $A_{0}$ (and $A_{1}$ ) is supersingular. We know that both $\Delta$ and $\operatorname{End}^{0}\left(A_{0}\right)$ have the same degree $\left(=2 \operatorname{dim} A_{1}\right)$ of maximal semi-simple commutative subalgebras. The centralizer of $K_{0}$ of $\operatorname{End}^{0}\left(A_{0}\right)$ is equal to the division algebra $\Delta$ and hence by bi-commutant theorem [11, Theorem 7.11 and Corollary 7.13, p. 94-95] that the centralizer of $\Delta$ in $\operatorname{End}^{0}\left(A_{0}\right)$ is equal to $K_{0}$. It follows that the center $Z$ of $\operatorname{End}^{0}\left(A_{0}\right)$ is contained in the totally real field $K_{0}$. By the classification of endomorphism algebras of abelian varieties over finite fields in Tate [13, the field $Z$ is equal to $\mathbb{Q}$ or $\mathbb{Q}(\sqrt{p})$ and $A_{0}$ is supersingular. We may enlarge the field $k_{0}$ so that $A_{0}$ is isogenous to the product of copies of supersingular elliptic curves with endomorphism algebra $D_{p, \infty}$. Therefore, $\operatorname{End}^{0}\left(A_{0}\right)=\operatorname{Mat}_{\left[K_{0}: \mathbb{Q}\right]}\left(D_{p, \infty}\right)$, noting that $\operatorname{dim} A_{0}=\operatorname{dim} A_{1}=g / n=\left[K_{0}: \mathbb{Q}\right]$. Then the division algebra $\Delta$ is equal to the centralizer of $K_{0}$ in $\operatorname{Mat}_{\left[K_{0}: \mathbb{Q}\right]}\left(D_{p, \infty}\right)$. It follows that $\Delta$ is ramified 
exactly at all Archimedean places and finite places $v$ of $K_{0}$ over $p$ of odd degree, or equivalently $\Delta \simeq D_{p, \infty} \otimes_{\mathbb{Q}} K_{0}$. If we assume the hypothesis $(\mathbf{H})$ (at the end of Section 21), then there are only two possibilities for $\Delta$ : either $\Delta=D_{p, \infty}$ or $\Delta=D_{\infty_{1}, \infty_{2}}$, the definite quaternion algebra over the field $\mathbb{Q}(\sqrt{p})$ which is ramified exactly at two Archimedean places $\infty_{1}$ and $\infty_{2}$. We have given all possibilities of the division algebras $\Delta$, and only the cases $\Delta=D_{p, \infty}$ and $\Delta=D_{\infty_{1}, \infty_{2}}$ can occur as endomorphism algebras of simple abelian varieties over finite fields.

We now show that the field $F$ contains a subfield which is isomorphic to $K_{0}$, so that we may assume that $F$ contains $K_{0}$ and that the embedding $\iota$ is a $K_{0}$-algebra homomorphism. Let $x \mapsto \bar{x}$ be the canonical involution of $\Delta$, which is the unique positive involution. Define a positive involution $*$ on $\operatorname{Mat}_{n}(\Delta)$ by $\left(a_{i j}\right)^{*}=\left(\overline{a_{j i}}\right)$. We know that for any embedding $\iota: F \rightarrow \operatorname{Mat}_{n}(\Delta)$, there is a positive involution $*_{1}$ which leaves the image $\iota(F)$ invariant and every element of $\iota(F)$ invariant. On the other hand, one can show that there is an isomorphism of algebras with involution $\left(\operatorname{Mat}_{n}(\Delta), *_{1}\right) \simeq\left(\operatorname{Mat}_{n}(\Delta), *\right)$. This follows from the Noether-Skolem theorem, the fact that the unitary group $U\left(\operatorname{Mat}_{n}(\Delta), *\right)$ is semi-simple and simply-connected, and the Kneser theorem on the $H^{1}$-vanishing for simply-connected groups over non-Archimedean local fields. For the details of this argument, see for example [17, Section 2]. Therefore, we may assume that the image of $F$ is fixed by $*$. Since the maximal semi-simple commutative subalgebras of $\operatorname{Mat}_{n}(\Delta)$ stable by the involution has degree $n\left[K_{0}: \mathbb{Q}\right]$ over $\mathbb{Q}$, the image $\iota(F)$ contains the center $K_{0}$. This shows that $F$ contains a subfield that is isomorphic to $K_{0}$.

As $\left[F: K_{0}\right]=n$, a $K_{0}$-algebra embedding $\iota: F \rightarrow \operatorname{Mat}_{n}(\Delta)$ always exists if $\Delta$ is the endomorphism algebra of a simple abelian variety $A_{1}$ of dimension $\left[K_{0}: \mathbb{Q}\right]$. The abelian variety $\left(A=A_{1}^{n}, \iota\right)$ with $\mathrm{RM}$ by $F$ has endomorphism algebra $\operatorname{End}^{0}(A) \simeq \operatorname{Mat}_{n}(\Delta)$.

(Type IV) The algebra $\Delta$ is a central simple algebra over a CM field $K$ with maximal real number field $K_{0}$. For any finite place $v$ of $K$, one has

$$
\operatorname{inv}_{v}(\Delta)+\operatorname{inv}_{\sigma(v)}(\Delta)=0, \quad \text { and } \quad \operatorname{inv}_{v}(\Delta)=0 \quad \text { if } \quad \sigma(v)=v,
$$

where $\sigma$ is the non-trivial automorphism of $K / K_{0}$. This is the necessary and sufficient condition for the central simple $\Delta$ that admits a positive involution. Let $m:=\operatorname{deg}(\Delta)$. The algebra $\Delta$ contains a (maximal) totally real subfield $F^{\prime}$ of degree $m\left[K_{0}: \mathbb{Q}\right]$ over $\mathbb{Q}$, therefore one has $m\left[K_{0}: \mathbb{Q}\right] \mid \operatorname{dim} A_{1}$, or equivalently $n m\left[K_{0}: \mathbb{Q}\right] \mid g$. On the other hand, since the field $F$ can be embedded into $\operatorname{Mat}_{n}(\Delta)$, one has the condition $g \mid n m\left[K_{0}: \mathbb{Q}\right]$. This shows $\operatorname{dim} A_{1}=m\left[K_{0}: \mathbb{Q}\right]$ and $A_{1}$ has smCM. Since any maximal semi-simple commutative subalgebra of $\operatorname{Mat}_{n}(\Delta)$ that is stable for a positive involution has degree $n m\left[K_{0}: \mathbb{Q}\right]$, which is also equal to $[F: \mathbb{Q}]$, the image $\iota(F)$ contains a subfield which is isomorphic to $K_{0}$. Therefore, we may assume that $F$ contains $K_{0}$ and that the embedding map $\iota$ is a $K_{0}$-algebra homomorphism.

We now determine the condition for $\Delta$ so that the totally real field $F$ can be embedded into $\operatorname{Mat}_{n}(\Delta)$ over $K_{0}$. Note that $\left[F: K_{0}\right]=\operatorname{deg}\left(\operatorname{Mat}_{n}(\Delta) / K\right)$. Put $L=F \otimes_{K_{0}} K$. The CM field $L$ is isomorphic to a maximal subfield of $\operatorname{Mat}_{n}(\Delta)$. The local-global principle (cf. [10, Theorem A.1] and [18) asserts that this holds if and only if for any (finite) ramified place $v$ of $K$ for $\Delta$, one has $\left[L_{w}: K_{v}\right] \cdot \operatorname{inv}_{v}(\Delta) \in \mathbb{Z}$. If $v$ is fixed by $\sigma$, then $\operatorname{inv}_{v}(\Delta)=0$ and hence the condition is satisfied automatically. 
Let $v$ be a finite ramified place of $K$ and $v_{0}$ be the place of $K_{0}$ below $v$; the place $v_{0}$ splits in $K$. For any place $w_{0}$ of $F$ lying over $v_{0}$, one also has that $w_{0}$ splits in $L$. Therefore $\left[F_{w_{0}}: K_{0, v_{0}}\right]=\left[L_{w}: K_{v}\right]$, where $w$ is any place of $L$ over $w_{0}$. One concludes that the field $F$ can be embedded into $\operatorname{Mat}_{n}(\Delta)$ over $K_{0}$ if and only if for any (finite) ramified place $v$ of $K$ for $\Delta$ (note: $\sigma(v) \neq v$ ),

$$
\left[F_{w_{0}}: K_{0, v_{0}}\right] \cdot \operatorname{inv}_{v}(\Delta)=0 \quad(\text { in } \mathbb{Q} / \mathbb{Z}), \quad \forall w_{0} \mid v_{0} .
$$

Conversely, suppose we are given a central division algebra $\Delta$ over a CM field $K$ with maximal totally real field $K_{0}$ that satisfies the local condition (3.1). Suppose also that (1) $K_{0}$ is contained in $F,(2) m n\left[K_{0}: \mathbb{Q}\right]=[F: \mathbb{Q}]$, where $m=\operatorname{deg}(\Delta / K)$, and (3) the condition (3.2) is satisfied. Then there is an abelian variety $(A, \iota)$ with $\mathrm{RM}$ by $F$ such that $\operatorname{End}^{0}(A) \simeq \operatorname{Mat}_{n}(\Delta)$. Indeed, we take a complex abelian variety $A_{1}$ of dimension $m\left[K_{0}: \mathbb{Q}\right]$ such that $\operatorname{End}^{0}\left(A_{1}\right) \simeq \Delta$. Above discussion shows that there is a $K_{0}$-algebra embedding $\iota: F \rightarrow \operatorname{Mat}_{n}(\Delta)$. Set $A:=A_{1}^{n}$, then the pair $(A, \iota)$ has the desired property.

We summarize the classification in the following theorem. This is a result of Chai 3. Lemma 6], while we make it more explicit about simple algebras that can actually occur as endomorphism algebras of RM abelian varieties over more general (unspecified) ground fields.

Theorem 3.2. Let $(A, \iota)$ be a g-dimensional abelian variety with $R M$ by $F$ over a field $k$. Then $\operatorname{End}^{0}(A) \simeq \operatorname{Mat}_{n}(\Delta)$ for a positive integer $n$ and a division algebra $\Delta$.

(Type I) The algebra $\Delta=K_{0}$ is a totally real number field. Then the field $K_{0}$ can be embedded as a subfield in $F$ with $n\left[K_{0}: \mathbb{Q}\right]=g$.

Conversely, given a subfield $K_{0}$ of $F$ of degree $d$, then there is an abelian variety $(A, \iota)$ with $R M$ by $F$ such that $\operatorname{End}^{0}(A) \simeq \operatorname{Mat}_{n}\left(K_{0}\right)$, where $n:=g / d$.

(Type II) The algebra $\Delta$ is a totally indefinite quaternion algebra over a totally real number field $K_{0}$. Then the field $K_{0}$ can be embedded as a subfield of $F$ with $2 n\left[K_{0}: \mathbb{Q}\right]=g$ and $F$ splits the quaternion algebra $\Delta$.

Conversely, given a subfield $K_{0}$ of $F$ with $2 n\left[K_{0}: \mathbb{Q}\right]=g$ for some positive integer $n$, and $\Delta$ an indefinite quaternion algebra over $K_{0}$ such that $F$ splits $\Delta$. Then there is an abelian variety $(A, \iota)$ with $R M$ by $F$ such that $\operatorname{End}^{0}(A) \simeq \operatorname{Mat}_{n}(\Delta)$.

(Type III) The algebra $\Delta$ is a totally definite quaternion algebra over a totally real number field $K_{0}$. Then the field $K_{0}$ can be embedded as a subfield of $F$ with $n\left[K_{0}: \mathbb{Q}\right]=g$. The characteristic of the base field $k$ is a prime $p>0$, and $A$ is supersingular. Moreover, we have $\Delta \simeq D_{p, \infty} \otimes_{\mathbb{Q}} K_{0}$. Under the assumption of the hypothesis $(\mathbf{H})$, we have $\Delta \simeq D_{p, \infty}$ with $K_{0}=\mathbb{Q}$, or $D_{\infty_{1}, \infty_{2}}$ with $K_{0}=\mathbb{Q}(\sqrt{p})$.

Conversely, suppose $\left(\Delta, K_{0}\right)$ is one of the above two cases and suppose that $K_{0}$ is contained in $F$. Then there exists an abelian variety $(A, \iota)$ with $R M$ by $F$ over a finite field such that $\operatorname{End}^{0}(A) \simeq \operatorname{Mat}_{n}(\Delta)$, where $n=g /\left[K_{0}: \mathbb{Q}\right]$.

(Type IV) The algebra $\Delta$ is a central simple algebra over a $C M$ field $K$ with maximal real number field $K_{0}$. Then the field $K_{0}$ can be embedded in $F$ with $g=n m\left[K_{0}: \mathbb{Q}\right]$ 
where $m=\operatorname{deg}(\Delta / K)$. For any finite ramified place $v$ of $K$ for $\Delta$, we have

$$
\left[F_{w_{0}}: K_{0, v_{0}}\right] \cdot \operatorname{inv}_{v}(\Delta)=0 \quad(\text { in } \mathbb{Q} / \mathbb{Z}), \quad \forall w_{0} \mid v_{0},
$$

where $v_{0}$ is the place of $K_{0}$ below $v$.

Conversely, let $\Delta$ be a central division algebra over a $C M$ field $K$ with maximal totally real field $K_{0}$ that admits a positive involution. Suppose that (1) $K_{0}$ is contained in $F,(2) m n\left[K_{0}: \mathbb{Q}\right]=[F: \mathbb{Q}]$ for some positive integer $n$, where $m=\operatorname{deg}(\Delta / K)$, and (3) the condition (3.3) is satisfied. Then there is an abelian variety $(A, \iota)$ with $R M$ by $F$ such that $\operatorname{End}^{0}(A) \simeq \operatorname{Mat}_{n}(\Delta)$.

\section{ACKNOWLEDGMENTS}

The author thanks C.-L. Chai for his constant support and encouragement, as well as his work [3] where the second part of this paper is based on. The author was partially supported by grants NSC 100-2628-M-001-006-MY4 and AS-99-CDAM01. Finally he thanks the referee for helpful suggestions on the organization that improve the presentation of this paper.

\section{REFERENCES}

[1] S. Baba and H. Granath, Primes of superspecial reduction for QM abelian surfaces. Bull. London Math. Soc. 40 (2008), no. 2, 311-318.

[2] J.-F. Boutot and H. Carayol, Uniformisation p-adique des courbes de Shimura: les théorèmes de Čerednik et de Drinfel'd. Courbes modulaires et courbes de Shimura (Orsay, 1987/1988). Astérisque No. 196-197 (1991), 45-158.

[3] C.-L. Chai, Every ordinary symplectic isogeny class in positive characteristic is dense in the moduli. Invent. Math. 121 (1995), 439-479.

[4] L. Dieulefait and V. Rotger, The arithmetic of QM-abelian surfaces through their Galois representations. J. Algebra 281 (2004), no. 1, 124-143.

[5] Yu. Manin, Theory of commutative formal groups over fields of finite characteristic. Russian Math. Surveys 18 (1963), 1-80.

[6] D. Mumford, Abelian Varieties. Oxford University Press, 1974.

[7] F. Oort, The isogeny class of a CM-type abelian variety is defined over a finite extension of the prime field. J. Pure Appl. Algebra 3 (1973), 399-408.

[8] F. Oort, Which abelian surfaces are products of elliptic curves? Math. Ann. 214 (1975), 35-47.

[9] F. Oort, Endomorphism algebras of abelian varieties. Algebraic geometry and commutative algebra, in honor of M. Nagata (1988), 469-502.

[10] G. Prasad and A. Rapinchuk, Computation of the metaplectic kernel. Inst. Hautes Études Sci. Publ. Math. 84 (1996), 91-187.

[11] I. Reiner, Maximal orders. London Mathematical Society Monographs, No. 5. Academic Press, London-New York, 1975, 395 pp.

[12] Sheng-Chi Shih, Tse-Chung Yang and C.-F. Yu, Embeddings of fields in simple algebras over global fields. arXiv:1108.0830, 22 pp. To appear in Asian J. Math..

[13] J. Tate, Endomorphisms of abelian varieties over finite fields. Invent. Math. 2 (1966), 134144.

[14] J. Tate, Classes d'isogenie de variétés abéliennes sur un corps fini (d'après T. Honda). Sém. Bourbaki Exp. 352 (1968/69). Lecture Notes in Math., vol. 179, Springer-Verlag, 1971.

[15] W. C. Waterhouse, Abelian varieties over finite fields. Ann. Sci. École Norm. Sup. (4) 2 (1969), 521-560.

[16] C.-F. Yu, The isomorphism classes of abelian varieties of CM-type. J. Pure Appl. Algebra 187 (2004) 305-319.

[17] C.-F. Yu, On the slope stratification of certain Shimura varieties. Math. Z. 251 (2005), 859873. 
[18] C.-F. Yu, Embeddings of fields into simple algebras: generalizations and applications. J. Algebra 368 (2012), 1-20.

[19] C. Xing, On supersingular abelian varieties of dimension two over finite fields. Finite Fields Appl. 2 (1996), no. 4, 407-421.

Institute of Mathematics, Academia Sinica and nCtS (Taipei Office), 6th Floor, Astronomy Mathematics Building, No. 1, Roosevelt Rd. Sec. 4, Taipei, Taiwan, 10617

E-mail address: chiafu@math.sinica.edu.tw 